\title{
Photon burst detection of single atoms in an optical cavity
}

\author{
M. L. Terraciano ${ }^{1 \star}$, R. Olson Knell', D. G. Norris ${ }^{1}$, J. Jing ${ }^{1 \star}$, A. Fernández ${ }^{2}$ and L. A. Orozco ${ }^{1 \dagger}$
}

\begin{abstract}
Many protocols in atomic physics and quantum information hinge on the ability to detect the presence of neutral atoms ${ }^{1-4}$. Up to now, two avenues have been favoured: the direct detection of spontaneously emitted photons using high-quality optics $^{5-7}$, or the observation of changes in light transmission through cavity mirrors due to strong atom-photon coupling ${ }^{8-11}$. Here, we present an approach that combines these two methods by detecting an atom in a driven cavity mode through the collection of spontaneous emission and forward scattering into an undriven, orthogonally polarized cavity mode. Moderate atom-cavity coupling enhances the signal, enabling the detection of multiple photons from the same atom. This real-time measurement can establish the presence of a single freely moving atom in less than $1 \mu s$ with more than $\mathbf{9 9 . 7 \%}$ confidence, using coincidence measurements to decrease the rate of false detections.
\end{abstract}

Direct detection of single atoms and molecules through the collection of resonance fluorescence requires excellent optics, very good background rejection and typical integration times of tens of milliseconds, even for trapped atoms ${ }^{5-7}$. Faster results are possible with fluorescence burst detection ${ }^{12}$, which looks for above-average count rates over short time intervals ${ }^{13,14}$; a recent example ${ }^{15}$ showed detection of freely falling atoms in $60 \mu \mathrm{s}$ using highly efficient mirrors and lenses. Alternatively, one can collect fluorescence in an optical cavity with the axis perpendicular to the driving laser, gaining the benefit of Purcell-enhanced emission into the cavity mode ${ }^{9-11}$. Experiments based on changes in cavity transmission, which require strong atom-cavity coupling ${ }^{8}$, have achieved singleatom detection times of $20 \mu$ s for moving atoms ${ }^{16,17}$, and as low as $10 \mu$ s for trapped atoms ${ }^{18,19}$.

All of these techniques gather data (photon flux) until a targeted confidence level is reached: detection of fluorescence requires the building up of a signal against background, whereas detection through cavity transmission requires the averaging of shot noise until a change in intensity level is discernible. The resultant signalto-noise and signal-to-background ratios set the probability of obtaining a false positive for atom detection.

Here, we present a new approach that achieves high-fidelity single-atom detection in a short time. We use a cavity with two modes of orthogonal linear polarization $(\mathrm{H}$ and $\mathrm{V})$, while driving the cavity on-axis with light of only one of these polarizations $(\mathrm{H})$, a technique introduced in ref. 20. With a weak magnetic field set parallel to the incident polarization, the light drives $\pi$ $(\Delta m=0)$ transitions in ${ }^{85} \mathrm{Rb}$ atoms traversing the cavity mode. An excited atom can return to the ground state in one of two ways: by emitting light of the same polarization $(\mathrm{H})$ through a spontaneous or stimulated emission transition that preserves the ground-state $m$ number $(\Delta m=0)$; or, by emitting light of circular polarization through a spontaneous emission transition that changes the ground-state $m$ number $(\Delta m= \pm 1)$, a fraction of which is collected in the orthogonal cavity mode (V) (see Fig. 1a). The detection of light from this mode is a clear signature of the presence of an atom, provided that light arising from cavity mirror birefringence or background scattering is sufficiently suppressed. We have shown that in our system there is a Purcell enhancement of spontaneous emission into this mode ${ }^{21}$.

Alternatively, we can increase the signal from the atoms by applying a magnetic field parallel to the cavity axis, while keeping the same polarization arrangement. The field causes a Faraday rotation of the drive (forward scattered radiation), such that the polarization vector gains a component in the orthogonal mode (see Fig. 1b). The cavity finesse enables the light to compound multiple rotations before escaping (see the Methods section for further details). We present the Faraday rotation data unless otherwise specified. Although exciting the atoms with a laser perpendicular to the cavity axis would reduce background counts, it would also prevent us from detecting the forward scattered light through Faraday rotation.

Our apparatus (Fig. 1c) consists of a Fabry-Perot cavity with a mirror separation of $2.2 \mathrm{~mm}$, a decay rate of $\kappa / 2 \pi=3.2 \times 10^{6} \mathrm{~s}^{-1}$, a finesse of 11,000 and a $\mathrm{TEM}_{00}$ mode waist of $50 \mu \mathrm{m}$. The cavity length is kept resonant with the $F=3 \rightarrow F^{\prime}=4$ transition of the $D_{2}$ line of ${ }^{85} \mathrm{Rb}$ at $780 \mathrm{~nm}$. The birefringence splitting of the two polarization modes is less than $500 \mathrm{kHz}$. The source of atoms is a magneto-optical trap (MOT) in vacuum, modified to produce a low-velocity atomic beam ${ }^{22}$ escaping through a 1.5 -mm-diameter hole in the retro-optics of one arm. The atomic beam intersects the cavity mode about $8 \mathrm{~cm}$ below the trapping region. The single-atom dipole coupling frequency for the driven cavity mode is $g / 2 \pi=1.5 \mathrm{MHz}$ for $\pi$-polarized light, and the total atomic decay rate is $\gamma_{\text {tot }} / 2 \pi=6 \times 10^{6} \mathrm{~s}^{-1}$, giving a single-atom cooperativity of $C_{1}=g^{2} / \kappa \gamma_{\text {tot }}=0.12$ and a saturation photon number of $n_{\text {sat }}=\gamma_{\text {tot }}^{2} / 3 g^{2}=5.3$. (For a more extensive description of the system, see ref. 21.)

Atoms enter the cavity in the $F=3$ ground state, as the MOT beams have both cooling and repumping. The typical velocity of the atoms is $20 \mathrm{~m} \mathrm{~s}^{-1}$, and they remain in the mode for almost $5 \mu \mathrm{s}$. This time is much longer than the radiative lifetime of the excited state $(\tau=26 \mathrm{~ns}$ ), so they may undergo several cycles of excitation and emission before leaving the cavity, producing a burst of photons. The parameters of the system prevent us from observing single-atom transits through a decrease in cavity transmission ${ }^{8}$.

One or two avalanche photodiodes (APDs) detect photons from the $\mathrm{V}$ mode, and their arrival times are recorded with a

\footnotetext{
1Joint Quantum Institute, Department of Physics, University of Maryland and National Institute of Standards and Technology, College Park, Maryland 20742-4111, USA, ${ }^{2}$ Centro de Optica e Información Cuántica, Departamento de Física, Universidad de Concepción, Concepción 4070386, Chile. *Present addresses: Naval Research Laboratory, Washington DC 20375, USA (M.L.T.); State Key Laboratory of Precision Spectroscopy, Department of Physics, East China Normal University, Shanghai 200062, P. R. China (J.J.). †e-mail: lorozco@umd.edu.
} 


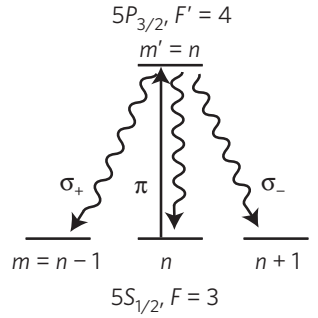

Atomic beam from LVIS

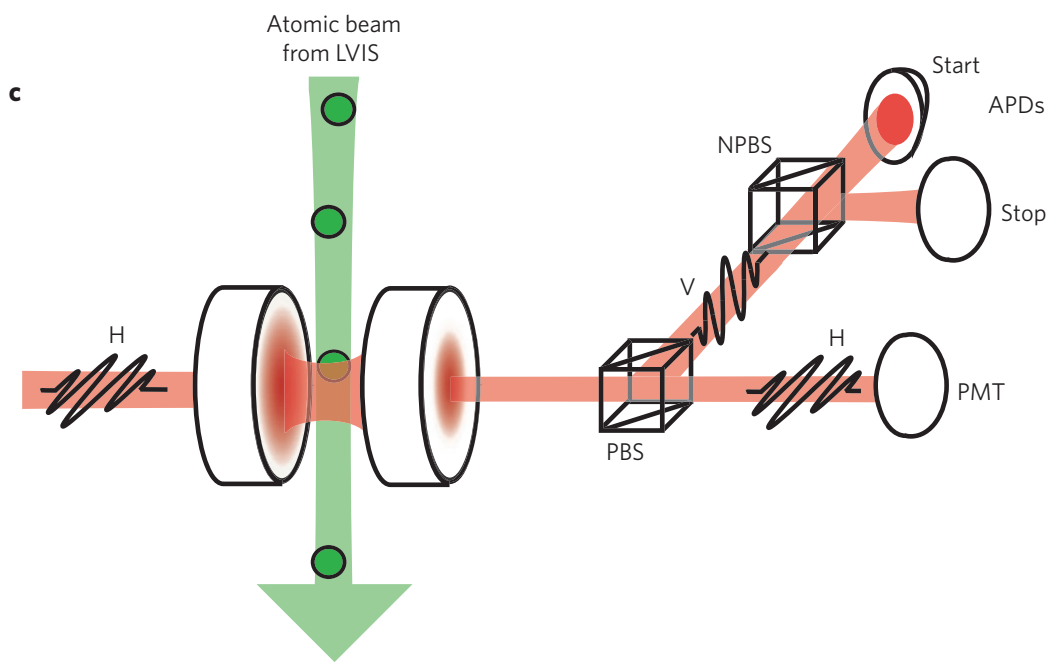

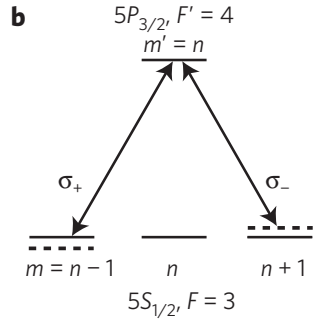

d

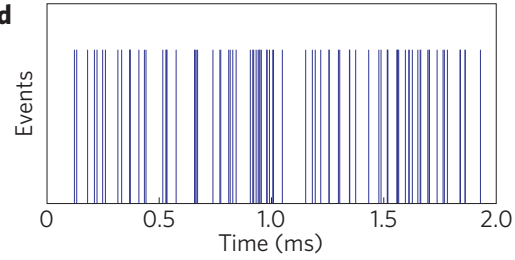

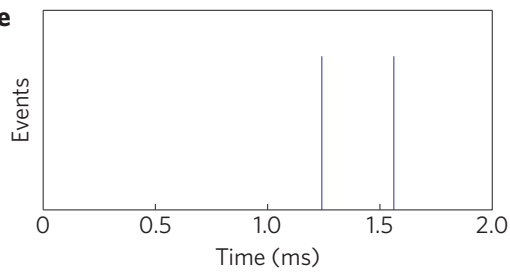

Figure 1 | Apparatus and detection scheme. a, Simplified diagram of ${ }^{85} \mathrm{Rb}$ levels relevant to spontaneous emission detection. A linearly polarized driving field excites the atom, but a transition to a ground-state level with different $m$ results in emission in the orthogonal polarization. $\mathbf{b}$, Simplified diagram of ${ }^{85} \mathrm{Rb}$ levels relevant to Faraday rotation in an applied magnetic field. The two circular components of a linearly polarized driving field accumulate a differential phase with ground-state Zeeman shift (dashed lines), resulting in a rotation of the output field polarization. c, Schematic diagram of the apparatus with the basic optical elements necessary for coincidence measurements. Horizontally polarized laser light $(\mathrm{H})$ drives the high-finesse cavity traversed by a beam of cold ${ }^{85} \mathrm{Rb}$ atoms from a low-velocity intense source (LVIS). The output light passes through a polarizing beam splitter (PBS) that separates the vertical $(V)$ polarization, sending it to a non-polarizing beam splitter (NPBS) for coincidence measurements using two avalanche photodiodes (APD). The $\mathrm{H}$ light is monitored by a photomultiplier tube (PMT). The output pulses from the APDs are counted in real time using a hardware counter and electronic gate to detect coincidences, or analysed offline from a time-stamped record of events. Not shown in the diagram is the direction of the magnetic field inside the cavity, which can be parallel to $\mathrm{H}$ ( $\pi$-polarized drive) or parallel to the direction of propagation ( $\sigma$-polarized drive). $\mathbf{d}$,e, Time series of recorded photon detections with (d) and without (e) atoms in the cavity, illustrating the high signal-to-background ratio in the $V$ mode. Atoms can populate the mode through spontaneous emission and Faraday rotation of the driving field. Without atoms, counts arise from background light, cavity birefringence and detector dark counts.

digital time-stamp (see Fig. 1d (with atoms) and Fig. 1e (without atoms)). Fluctuations in photon number in excess of those expected for a Poisson process give information about the number of photons scattered from each atom that passes through the cavity. We use the formula of Mandel relating the photon number distribution $P(n)$ to the atom number distribution $P_{\text {atom }}(m)$ : $P(n)=\sum_{m} P(n \mid m) P_{\text {atom }}(m)$, where $P(n \mid m)=(\alpha m)^{n} \exp (-\alpha m) / n$ ! is the conditional probability of detecting $n$ photons when there are $m$ atoms in the cavity volume, each contributing a mean of $\alpha$ photons to the signal with a Poisson distribution of number ${ }^{23}$. We follow the method of ref. 9 and use the above relations to write:

$$
\frac{\left\langle n^{2}\right\rangle}{\langle n\rangle}-1=g_{\text {aa }}\langle n\rangle+\alpha
$$

where $g_{\text {aa }}$ is the atom-atom correlation function; $g_{\text {aa }}=1$ if $P_{\text {atom }}(m)$ is also a Poisson distribution. We extract from the record of APD pulses the mean and mean-square photon number as a function of time bin size. The plot of equation (1) becomes linear for counting times longer than the transit time of the atoms through the cavity mode. Figure 2 shows the extracted values of $\alpha$, with changes in the cavity photon number (driving intensity) of more than an order of magnitude. The inset shows a plot of equation (1) for 3.5 photons
$(34 \mathrm{pW})$ in the driven mode of the cavity. A linear fit for bins from 50 to $100 \mu$ sgives a vertical axis intercept of $\alpha=0.267 \pm 0.003$ photons detected per atom, and a slope of $1.030 \pm 0.001$, indicating a mostly Poissonian source of atoms. The measured count rates from the $\mathrm{V}$ mode and the extracted values of $\alpha$ give an atomic flux of approximately 160,000 atoms $\mathrm{s}^{-1}$.

Detector dark counts (approximately $300 \mathrm{~s}^{-1}$ each) and counts from background light are present in the photon record in addition to the signal counts. At low driving intensity, the background count rate is dominated by scattered light coming from the MOT, but at higher intensity, light in the $\mathrm{V}$ mode arising from cavity birefringence (less than $4 \times 10^{-5}$ ) starts to degrade the signal-tobackground ratio. Figure 3 (filled squares) shows the measured ratio of signal count rate to background count rate as a function of driving intensity. The ratio is maximal for 3.5 photons in the $\mathrm{H}$ mode $\left(n / n_{\text {sat }}=0.67\right)$.

We could identify a single photon escaping from the $\mathrm{V}$ mode as evidence of an atom in the cavity; however, we would be wrong about $4 \%$ of the time owing to the background counts. We can suppress the probability of a false detection markedly by using the burst detection technique, and requiring photon coincidences in a time window smaller than the transit time of an atom across the cavity mode ${ }^{24}$. We implement this in real time using an electronic 


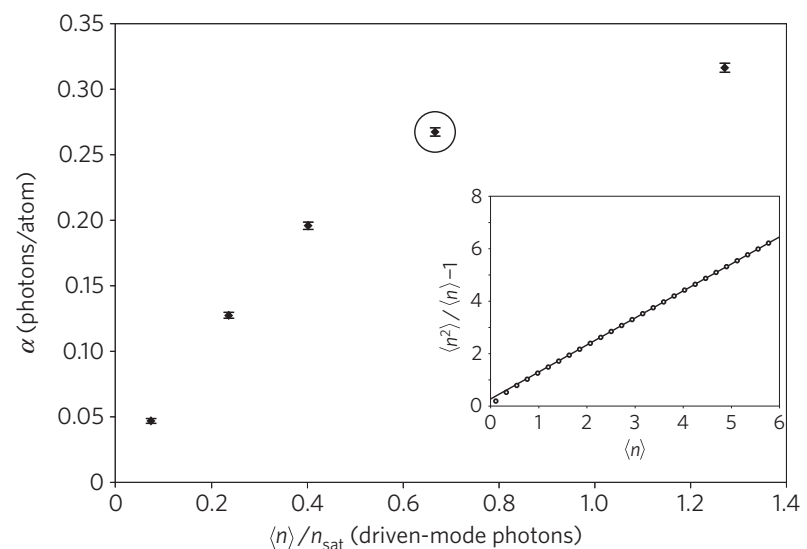

Figure 2 | Counts detected from a single atom. A statistical analysis of photon events from the $V$ mode enables us to extract the mean number of photons $\alpha$ detected from each atom passing through the cavity mode, assuming Poissonian fluctuations in atom number. The horizontal axis gives the intensity of the exciting laser, as measured by the steady-state intracavity photon number. As expected, $\alpha$ increases linearly with drive until atomic saturation intensity $\left(n_{\text {sat }}=5.3\right.$ photons). The circle indicates our operating point for maximum fidelity of detection. Error bars show \pm 1 s.d. in the determination of $\alpha$ for each point, given the number of counts used to determine $\langle n\rangle$ and $\left\langle n^{2}\right\rangle$. Inset: least-squares fit to equation (1) at the operating point, showing a vertical axis intercept of $\alpha=0.267(3)$.

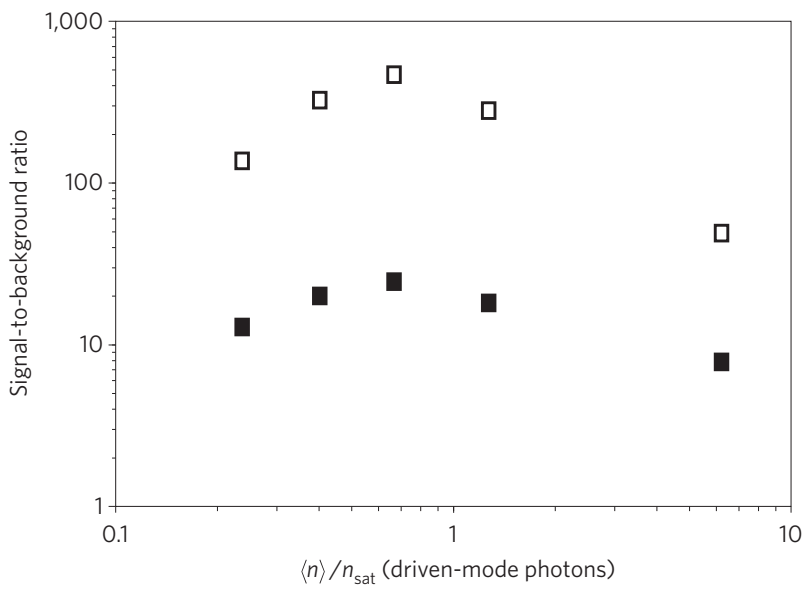

Figure 3 | Reducing background noise through coincidence detection of photons. Plot of the measured ratio of event rates in the $\mathrm{V}$ mode with and without atoms (signal-to-background ratio) as a function of driving intensity. Requiring two or more photons within a $1 \mu \mathrm{s}$ window for an event (open squares) improves the ratio by more than an order of magnitude compared with classing detection of single photons as the events (filled squares). Both sets achieve their maxima just before the atomic saturation intensity, owing to background counts from cavity birefringence that continue to increase linearly with drive. Standard deviations for the number of events collected are smaller than the size of the squares.

coincidence counter. The first vertically polarized photon detected by the 'start' APD opens a gate of variable width that enables passage of subsequent output pulses from the 'stop' APD. Figure 3 (open squares) shows the signal-to-background ratio for the rate of coincidence events in a $1 \mu$ s window. The maximum occurs at the same driving intensity for both singles and coincidence detection, but the latter is larger by more than an order of magnitude.

Figure $4 \mathrm{a}$ shows the extracted probability from a $300 \mathrm{~s}$ time series at $n / n_{\text {sat }}=0.67$ for $n$ counts in any interval of $1 \mu$ s. Requiring a two-photon coincidence is sufficient to increase the
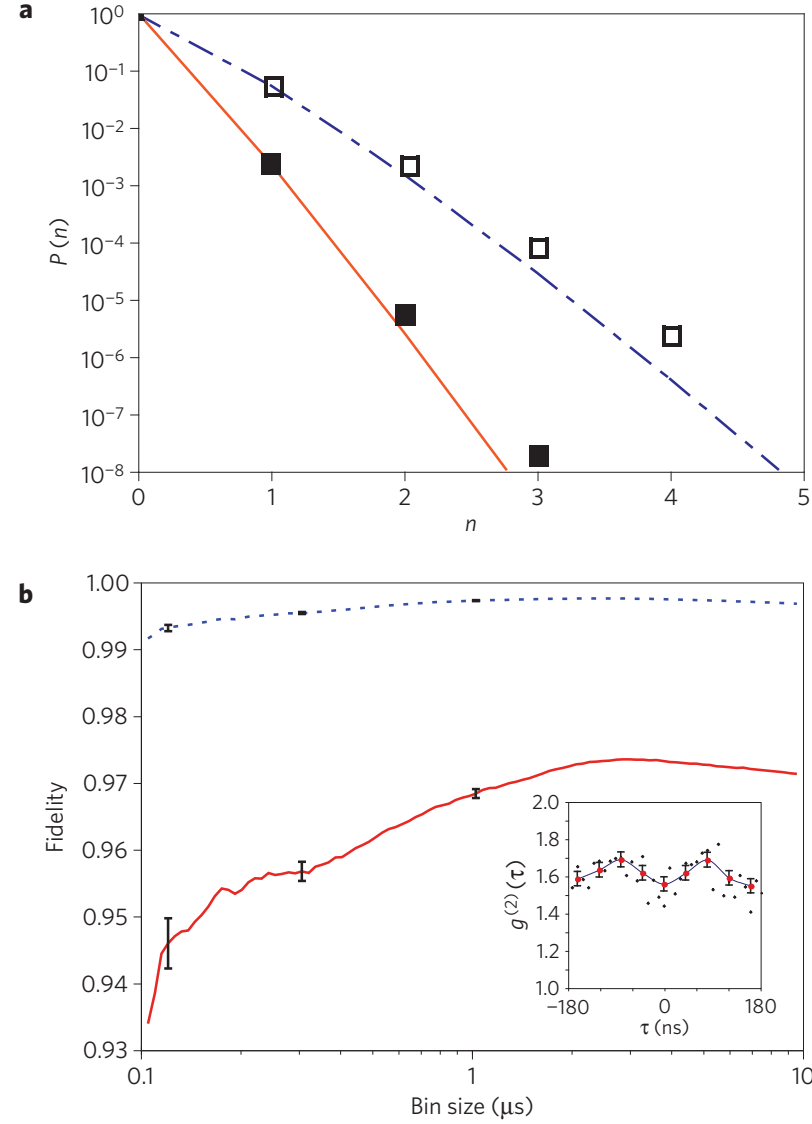

Figure 4 | Evaluation of the detection scheme. a, Measured probability of detecting $n$ photons in $1 \mu$ s with (open squares) and without (filled squares) atoms. The rapid divergence of the two series on a logarithmic scale shows the benefit gained from requiring $n>1$ for a detection event. The lines show the prediction for a Poisson distribution with the same mean number. Standard deviations for the number of events collected are smaller than the size of the squares. $\mathbf{b}$, Fidelity of atom detection based on two-photon coincidence as a function of coincidence gate length, on a logarithmic horizontal scale. The fidelity (one minus the probability of error) reaches more than $99 \%$ at $0.1 \mu \mathrm{s}$, and $99.7 \%$ at $1 \mu \mathrm{s}$ with Faraday rotation (blue dotted line), but only $96.7 \%$ at $1 \mu$ s with detection of spontaneous emission alone (red solid line) (plot $\mathbf{b}$ had $0.4 n / n_{\text {sat }}$ drive). Inset: short-time intensity autocorrelation function for the same atomic beam density under weak drive $\left(n / n_{\text {sat }}<0.1\right)$, measured between two APDs to circumvent detector dead time. The dip at equal times (photon antibunching) is of the order of the excited-state lifetime (26 ns) and indicates the dominance of single-atom interactions within the cavity. Small points represent data collected in 4 ns counting bins. Large points represent coarser division of the data into $40 \mathrm{~ns}$ bins, and are joined by a smooth curve as a visual aid. Error bars in $\mathbf{b}$ show \pm 1 s.d. for the number of counts collected in each time bin.

signal-to-background ratio by more than an order of magnitude, whereas requiring a three-photon coincidence reduces the probability of detection by another order of magnitude. Operating under a two-photon detection scheme, we extract the fidelity of detection $F$ versus gate length by integrating the waiting time distribution of photon arrivals on a single APD to obtain the number of two-photon coincidences $c$ in a given time window. Doing this for time series with and without atomic flux, we calculate $F=1-c_{\text {without }} / c_{\text {with }}$. The fidelity reaches $99.43 \pm 0.02 \%$ for a time window of $0.2 \mu$ s and $99.719 \pm 0.006 \%$ in $1 \mu$ s (see Fig. $4 b$ ) when using Faraday rotation (blue dotted line). For comparison, we also plot the fidelity relying only on spontaneous emission into 
the $\mathrm{V}$ mode (red solid line), which reaches $96.70 \pm 0.07 \%$ in $1 \mu \mathrm{s}$, indicating more than a factor of ten greater probability of a false detection. The difference is a result of the four times greater signal-to-background ratio for singles count rates using the Faraday rotation. The fidelity is optimal for times between 1 and $5 \mu \mathrm{s}$, determined by the distribution of atom transit times.

To ensure that our coincidence detection scheme is sensitive to single atoms, we measure the intensity autocorrelation function $\left(g^{(2)}(\tau)\right)$ of the $\mathrm{V}$ mode under very weak driving intensity $\left(n / n_{\text {sat }}<0.1\right)$ at the same atomic beam density used during detection measurements. We observe photon antibunching with a time width of the order of the excited-state lifetime (26 ns) (see inset in Fig. 4b). This signal decreases as the inverse of the mean number of atoms in the cavity ${ }^{25}$, and we have verified that it disappears in our system at even moderately higher atomic beam densities. As the antibunching signal in resonance fluorescence can arise only from photon pairs emitted from the same atom, this confirms our ability to detect coincidence counts from single atoms in less than $1 \mu \mathrm{s}$. On the basis of the measured rate of atomic flux and mean transit time through the mode $(4.3 \mu \mathrm{s})$, we obtain a mean atom number of 0.69 in the cavity volume. This yields a $15 \%$ probability of having more than one atom in the detection region at any time, given Poissonian fluctuations in number. However, the probability of having more than one well-coupled atom is much smaller: the measured drop in transmission of the driven cavity mode at this density is about $1 \%$, corresponding to an average of 0.05 maximally coupled atoms for our cavity parameters. At this density and using the methods of ref. 26, we calculate a probability of $10.8 \%$ for finding between 0.1 and 1 effective maximally coupled atoms, but only a $0.2 \%$ chance of finding an effective number greater than 1. A sharp decrease in probability above 1 effective atom indicates that we operate in a density regime of single-atom coupling, with a low probability of exciting two atoms simultaneously.

Detection efficiency and fidelity can be improved through stronger atom-cavity coupling (collecting more of the spontaneous emission) or higher cavity finesse (yielding a larger Faraday rotation). We anticipate that the application of this technique will be of particular use in cavity quantum electrodynamics research, where it is often preferable to integrate the output signal from a cavity only when one is sure that there are atoms coupled to the mode. The shortness of the detection window compared with the transit time leaves open the possibility of data collection triggered on a real-time detection event.

\section{Methods}

Faraday rotation. The detection of light from the orthogonally polarized cavity mode that lies at the heart of our detection scheme is enhanced significantly in the presence of a magnetic field parallel to the direction of light propagation (the Faraday effect). This cross-polarizer technique suppresses the driving light by many orders of magnitude, enabling the study of the forward scattered radiation ${ }^{27}$ We treat this effect in our system as a strong drive in one polarization and an orthogonal part consisting of two components: spontaneous emission and forward scattering. The spontaneous emission part quantitatively explains the count rates that we observe in the absence of Faraday rotation ${ }^{21}$. The increase in count rate in the presence of the longitudinal magnetic field is a Faraday rotation of the drive (forward scattering).

We consider an atom in free space and a cavity that effectively increases the number of interactions of the light with the atom. We simplify the atomic structure to one excited state $\left(m^{\prime}=0\right)$ and three ground states $(m= \pm 1,0)$. A magnetic field along the direction of light propagation lifts the ground-state degeneracy through the Zeeman effect, resulting in a shift in the dispersion curves and a difference in the phase velocities of the two circular components of light ${ }^{27}$ (see Fig. 1b). We excite the system with horizontally polarized light, which is an equal superposition of right- and left-circularly polarized light. In general, the transmitted light will become elliptically polarized, and the plane of polarization will rotate through an angle

$$
\phi=\frac{2 g_{\mathrm{L}} \mu_{\mathrm{B}} B / \hbar \gamma_{\text {tot }}}{1+\left(2 g_{\mathrm{L}} \mu_{\mathrm{B}} B / \hbar \gamma_{\text {tot }}\right)^{2}} C_{1}
$$

where $g_{\mathrm{L}}$ is the Landé factor, $\mu_{\mathrm{B}}$ is the Bohr magneton and $C_{1}$ is the single-atom cooperativity, which is related to the absorption length times the number of passes the light makes through the atom due to the cavity mirrors. We calculate the expected rotation for the ${ }^{85} \mathrm{Rb} F=3$ ground state $\left(g_{\mathrm{L}}=1 / 3\right.$ and $\mu_{\mathrm{B}} / h=1.4 \mathrm{MHz}$ per gauss) at $B=3$ gauss and obtain a rotation angle of $\phi=0.045 \pm 0.01 \mathrm{rad}$. To evaluate the rotation experimentally, we use the Stokes parameter formalism ${ }^{28}$, which enables a measurement of $\phi$ if the amplitudes of the electric field in the two polarizations $\varepsilon_{\perp}$ and $\varepsilon_{\|}$are known:

$$
|\phi|=\frac{\varepsilon_{\|} \varepsilon_{\perp}}{\left|\varepsilon_{\|}\right|^{2}-\left|\varepsilon_{\perp}\right|^{2}} \approx \frac{\varepsilon_{\perp}}{\varepsilon_{\|}}
$$

From the measured increase in the count rate in the single-atom regime, we obtain $\phi=0.038 \mathrm{rad}$, in good agreement with the simplified model. Unequal $m$ sublevel populations influence the behaviour in the real system.

Cavity stabilization and detunings. The input and output mirrors of the Fabry-Perot cavity have different transmission coefficients, with the output a factor of ten larger than the input, creating an asymmetric cavity biased towards the output. The cavity length is stabilized by the Pound-Drever-Hall method with light derived from an auxiliary laser at $820 \mathrm{~nm}$. A grating and interference filters separate the $780 \mathrm{~nm}$ signal light and $820 \mathrm{~nm}$ locking light on the cavity output. We find the peak count rate for the orthogonal mode with Faraday rotation by scanning the laser and cavity together across the atomic resonance. We take all measurements at the location of the peak, which is shifted by about $3 \mathrm{MHz}$ from the $F=3, m=0 \rightarrow F^{\prime}=4, m^{\prime}=0$ transition.

Counting and photon statistics. Each point in Fig. 2 was calculated from a $300 \mathrm{~s}$ record of photon events from the orthogonal (V) cavity mode onto a single APD, and corresponds to the vertical axis intercept of a least-squares fit to equation (1) for counting intervals between $\langle n\rangle=2.72$ and $\langle n\rangle=5.45$ (50-100 $\mu \mathrm{s})$. The calculated values include the $23 \%$ measured photon detection efficiency for the APD and optical path. An extra $300 \mathrm{~s}$ series was recorded independently for each point with the atom source turned off for background studies, which showed that residual noise from cavity birefringence distorts the numbers at higher driving intensities. These points were excluded from the plot.

The open squares in Fig. 3 were calculated by computer from the same $300 \mathrm{~s}$ time series as above to simulate real-time coincidence detection with an electronic gate and hardware counter. Detector after-pulsing contributed a small number of coincidences $(<1 \%)$, but did not significantly affect the results. Driven-mode intensities were varied over a factor of 26 , and single-photon count rates ranged from 22,600 to $98,400 \mathrm{~s}^{-1}$

The probabilities and fidelities in Fig. 4 were calculated from the same time series as above, with an extra $300 \mathrm{~s}$ series taken without Faraday rotation. The inset $g^{(2)}$ was calculated from a separate time series in which the V mode output was divided between two APDs with a 50/50 beam splitter. This prevents distortion from the $50 \mathrm{~ns}$ detector dead time and after-pulsing probability.

Received 13 February 2008; accepted 17 April 2009; published online 17 May 2009

\section{References}

1. Cirac, J. I., Zoller, P., Kimble, H. J. \& Mabuchi, H. Quantum state transfer and entanglement distribution among distant nodes in a quantum network. Phys. Rev. Lett. 78, 3221-3224 (1997).

2. Gheri, K. M., Saavedra, C., Törmä, P., Cirac, J. I. \& Zoller, P. Entanglement engineering of one-photon wave packets using a single-atom source. Phys. Rev. A 58, R2627-R2630 (1998).

3. Brennen, G. K., Caves, C. M., Jessen, P. S. \& Deutsch, I. H. Quantum logic gates in optical lattices. Phys. Rev. Lett. 82, 1060-1063 (1999).

4. Schön, C., Solano, E., Verstraete, F., Cirac, J. I. \& Wolf, M. M. Sequential generation of entangled multiqubit states. Phys. Rev. Lett. 95, 110503 (2005).

5. Schlosser, N., Reymond, G., Protsenko, I. \& Grangier, P. Sub-poissonian loading of single atoms in a microscopic dipole trap. Nature 411, 1024-1027 (2001)

6. Kuhr, S. et al. Deterministic delivery of a single atom. Science $\mathbf{2 9 3}$, 278-280 (2001)

7. Jones, M. P. A. et al. Fast quantum state control of a single trapped neutral atom. Phys. Rev. A 75, 040301 (R) (2007).

8. Mabuchi, H., Turchette, Q. A., Chapman, M. S. \& Kimble, H. J. Real-time detection of individual atoms falling through a high-finesse optical cavity. Opt. Lett. 21, 1393-1395 (1996).

9. Teper, I., Lin, Y.-J. \& Vuletić, V. Resonator-aided single-atom detection on a microfabricated chip. Phys. Rev. Lett. 97, 023002 (2006).

10. Nussmann, S. et al. Vacuum-stimulated cooling of single atoms in three dimensions. Nature Phys. 1, 122-125 (2005).

11. Fortier, K. M., Kim, S. Y., Gibbons, M. J., Ahmadi, P. \& Chapman, M. S. Deterministic loading of individual atoms to a high-finesse optical cavity. Phys. Rev. Lett. 98, 233601 (2007). 
12. Lewis, D. A., Tonn, J. F., Kaufman, S. L. \& Greenlees, G. W. Photon-burst method in high-resolution laser spectroscopy. Phys. Rev. A 19 , 1580-1588 (1979).

13. Peck, K., Stryer, L., Glazer, A. N. \& Mathies, R. A. Single molecule fluorescence detection: Autocorrelation criterion and experimental realization with phycoerythrin. Proc. Natl Acad. Sci. USA 86, 4087-4091 (1989).

14. Lee, Y.-H., Maus, R. G., Smith, B. W. \& Winefordner, D. Laser-induced fluorescence detection of a single molecule in a capillary. Anal. Chem. 66, 4142-4149 (1994).

15. Bondo, T., Hennrich, M., Legero, T., Rempe, G. \& Kuhn, A. Time-resolved and state-selective detection of single freely falling atoms. Opt. Commun. 264, 271-277 (2006).

16. Öttl, A., Ritter, S., Köhl, M. \& Esslinger, T. Correlations and counting statistics of an atom laser. Phys. Rev. Lett. 95, 090404 (2005).

17. Trupke, M. et al. Atom detection and photon production in a scalable, open, optical microcavity. Phys. Rev. Lett. 99, 063601 (2007).

18. Puppe, T. et al. Trapping and observing single atoms in a blue-detuned intracavity dipole trap. Phys. Rev. Lett. 99, 013002 (2007).

19. Boozer, A. D., Boca, A., Miller, R., Northup, T. E. \& Kimble, H. J. Cooling to the ground state of axial motion for one atom strongly coupled to an optical cavity. Phys. Rev. Lett. 97, 083602 (2006).

20. Birnbaum, K. M. et al. Photon blockade in an optical cavity with one trapped atom. Nature 436, 87-90 (2005).

21. Terraciano, M. L. et al. Enhanced spontaneous emission into the mode of a cavity QED system. Opt. Lett. 32, 982-984 (2007).
22. Lu, Z. T. et al. Low-velocity intense source of atoms from a magneto-optical trap. Phys. Rev. Lett. 77, 3331-3334 (1996).

23. Wilzbach, M. et al. A simple integrated single-atom detector. Opt. Lett. 34 259-261 (2009)

24. Aoki, T. et al. Observation of strong coupling between one atom and a monolithic microresonator. Nature 443, 671-674 (2006).

25. Hennrich, M., Kuhn, A. \& Rempe, G. Transition from antibunching to bunching in cavity QED. Phys. Rev. Lett. 94, 053604 (2005).

26. Carmichael, H. J. \& Sanders, B. C. Multiatom effects in cavity QED with atomic beams. Phys. Rev. A 60, 2497-2504 (1999).

27. Budker, D. et al. Resonant nonlinear magneto-optical effects in atoms. Rev. Mod. Phys. 74, 1153-1201 (2002).

28. Labeyrie, G., Miniatura, C. \& Kaiser, R. Large Faraday rotation of resonant light in a cold atomic cloud. Phys. Rev. A 64, 033402 (2001).

\section{Acknowledgements}

This work was supported by NIST and NSF. We are grateful to PicoQuant Photonics for their loan of the PicoHarp 300 time-correlated single photon counting module.

\section{Additional information}

Reprints and permissions information is available online at http://npg.nature.com/ reprintsandpermissions. Correspondence and requests for materials should be addressed to L.A.O. 